\title{
Implementación de actividades colaborativas a través de WhatsApp para incrementar vocabulario en inglés
}

\section{Collaborative activities implementation through WhatsApp to increase English vocabulary}

Wilson Gonzalo Rojas Yumisaca. ${ }^{1}$, Cristina Paola Chamorro Ortega. ${ }^{2}$, Silvia Nataly Bejarano Criollo. ${ }^{3}$

Recibido: 10-09-2019 / Revisado: 01-10-2019 /Aceptado: 15-10-2019/ Publicado: 04-11-2019

\begin{abstract}
DOI: https://doi.org/10.33262/cienciadigital.v3i4.1.983
\end{abstract}

This research responds to the need to innovate the teaching-learning process of the English as a Foreign Language through the implementation of technology and together with the use of a social application in the classrooms, specifically with the use of WhatsApp application, considering that this application was not designed for educational or academic purposes, but today it has a great acceptance and use. The main objective is that students with the help of the most uses mobiles devices and the WhatsApp application, increasing students' knowledge of vocabulary in English language. This quasi-experimental research was developed at the level of the Unified General Baccalaureate. For data collection, tabulation and analysis of results both descriptive and inferential statistics were used. The initial survey which was conducted to the entire population highlights through the results obtained the relevance that technology has today in collaborative learning, while the result of the pre-test and post-

${ }^{1}$ Escuela Superior Politécnica de Chimborazo, Facultad de Informática y Electrónica. Riobamba, Ecuador., wilson.rojas@espoch.edu.ec

2 Escuela Superior Politécnica de Chimborazo, Facultad de Recursos Naturales. Riobamba, Ecuador., cristina.chamorro@espoch.edu.ec

${ }^{3}$ Escuela Superior Politécnica de Chimborazo, Facultad de Administración de Empresas. Riobamba, Ecuador, sbejarano@espoch.edu.ec 
test affirmed the effectiveness of the use of WhatsApp in improving vocabulary in the English language as a foreign language.

Keywords: Collaborative Learning, English as a Foreign Language, Technology, WhatsApp, Vocabulary.

\section{Resumen.}

La presente investigación responde a la necesidad de innovar el proceso de enseñanzaaprendizaje del idioma inglés como lengua extranjera a través de la implementación de tecnología y junto al uso de dispositivos móviles en el aula de clases de inglés, específicamente con el uso de la aplicación social WhatsApp, considerando que esta aplicación no fue diseñada con propósitos académicos educativos, pero hoy en día tiene una gran aceptación y uso. El objetivo principal es que los estudiantes con la ayuda de los dispositivos móviles más utilizados y la aplicación WhatsApp, incrementen su conocimiento de vocabulario en el idioma inglés. Esta investigación fue de tipo cuasi experimental la cual se desarrolló en el nivel de Bachillerato General Unificado. Para la recolección, tabulación y análisis de resultados se empleó tanto estadística descriptiva como estadística inferencial. La encuesta inicial que se realizó a toda la población resaltó a través de los resultados obtenidos la relevancia que hoy en día tiene el uso de la tecnología en el aprendizaje colaborativo, mientras que el resultado de las pruebas pre - test y post - test afirmaron la eficacia del uso de la aplicación WhatsApp en el mejoramiento del léxico en el idioma inglés como lengua extranjera

Palabras claves: Aprendizaje Colaborativo, Inglés como Lengua Extranjera, Tecnología, WhatsApp, Vocabulario.

\section{Introducción.}

Esta investigación se enfoca en la implementación de actividades colaborativas a través de la aplicación social WhatsApp en dispositivos móviles más utilizados por los estudiantes con el objetivo principal de incrementar palabras nuevas en inglés en los estudiantes de primero, segundo y tercer Año de Bachillerato General Unificado de la Unidad Educativa "San Gerardo" de la ciudad de Riobamba.

De acuerdo a un estudio llevado a cabo por el IAB (Interactive Advertising Bureau) el 18 de diciembre de 2017, 90\% de los usuarios de redes sociales utilizan WhatsApp como una herramienta con diversos fines y propósitos entre ellos el más principal la comunicación (Arboleda, 2017). 
De igual manera, en un reporte realizado por el diario el telégrafo, de fecha 29 de julio de 2017, el número de usuarios quienes tienen acceso y utilizan como la aplicación más barata y de fácil uso es WhatsApp, la misma que asciende a 1000 millones al día de usuarios (Telégrafo, 2017).

Sin embargo, la encuesta aplicada a 69 alumnos de la Unidad Educativa "San Gerardo" demuestra que a pesar de que el $62,5 \%$ de los encuestados utilizan celulares, únicamente el $2,9 \%$ de la población ha utilizado aplicaciones que fomenten el aprendizaje de inglés y el $4,3 \%$ ha desarrollado actividades para mejorar su vocabulario. Esto resalta que existe un porcentaje menor en utilizar tanto los dispositivos como también las aplicaciones para el incremento de vocabulario.

Por otra parte, Juana Rubio (2015), en su artículo señala que la aplicación WhatsApp como una de las principales vías de acceso a comentarios son las redes sociales $(66,6 \%)$ y WhatsApp $(42,6 \%)$, lo cual nos permite notar que una de las principales aplicaciones más útiles que hace eco a la brecha del uso de la tecnología junto al uso del internet en los celulares móviles es WhatsApp. (Rubio, 2015).

De igual forma María Calero (2014) en su artículo menciona que el WhatsApp es una aplicación con grandes ventajas comunicativas, tecnológicas y económicas, pero también con sus defectos lo que está derivando en la rápida aparición de vías alternativas y aun así la aplicación WhatsApp les interesa como lingüistas por haberse convertido en un testimonio más de capacidad de adaptación y colonización de la competencia comunicativa humana en cualquier medio, lo cual nos manifiesta que la aplicación WhatsApp puede aportar académicamente por todas sus bondades pero siendo mal utilizada no dejaría de ser un sistema de mensajería social para lo cual fue creada (Calero, 2014)

La dependencia de los dispositivos móviles es una realidad que se debe aprovechar para el aprendizaje a fin de responder a la demanda educativa del Siglo XXI, ya que hoy en día la mayoría de estudiantes tiene acceso a el uso de celulares. (Basantes, 2017).

Los estudios más relevantes sobre la utilización de la aplicación WhatsApp para aprendizaje de vocabulario en inglés, se mencionan a continuación. El primero titulado "El uso del teléfono móvil y la aplicación WhatsApp para el aprendizaje de verbos frasales ingleses en alumnos de nivel intermedio" presentado por Raquel Calderó Gené, menciona que la incorporación del teléfono móvil como recurso didáctico es motivadora y atractiva; además, brinda la posibilidad de adquirir vocabulario en general con la guía adecuada para obtener un aprendizaje significativo (Caldero, 2014).

Por otra parte, los investigadores Lenín Lara, Marcelo Aullauca, Lorena Yumi, Carmen Mejía, Carmita Rojas y Mónica Logroño, en su artículo científico "Uso didáctico de WhatsApp para desarrollar la participación de los estudiantes en el aprendizaje de lenguas 
extranjeras" concluyen que WhatsApp ofrece una infinidad de opciones para el aprendizaje ya que esta aplicación facilita la interacción entre la comunidad académica y por tanto la participación del estudiante superando de esta manera las barreras de tiempo y espacio. En el aprendizaje de las lenguas extranjeras, WhatsApp resulta particularmente una aplicación útil, pues facilita la participación a través del trabajo interactivo, momentáneo y de fácil acceso entre la comunicación docente-estudiante y se mantiene el control aprovechando una guía y uso adecuado de todos sus integrantes al estar bajo parámetros y reglas establecidas. (Lenin Lara, 2017).

Finalmente, y a fin de enfatizar el aspecto colaborativo del uso del WhatsApp, se observa el estudio "Aprendizaje Colaborativo a través de WhatsApp y Facebook" en el cual María Abigail Sánchez Ramírez (2017), enfatiza que la importancia del uso de nuevas tecnologías, especialmente los dispositivos móviles, facilitan el trabajo y aprendizaje colaborativo, puesto que estos permiten a los estudiantes compartir información, trabajar con documentos conjuntos y que además facilitan la solución de problemas (RAMÍREZ, 2017)

Las ventajas de aprovechar el uso de celulares en el proceso de enseñanza-aprendizaje son mayores de las que imaginamos, el conocimiento, uso y habilidad en su manejo es algo que los docentes debemos aprovechar a nuestro favor. Ya que, aún prevalece la educación tradicional, este es el caso de la Unidad Educativa "San Gerardo" donde el profesor sigue siendo el único responsable del aprendizaje, es quien define los objetivos, las tareas y evalúa lo que los estudiantes han aprendido. Por esta razón, se recomienda a los docentes generar clases colaborativas que favorezcan las relaciones interpersonales donde la autoridad sea compartida con los estudiantes, conformando grupos en WhatsApp (Recursos Educativos, 2016)

Por lo anteriormente expuesto, el objetivo principal de este trabajo de investigación es implementar y promover las actividades colaborativas a través de la aplicación WhatsApp para la adquisición de vocabulario en el idioma inglés como lengua extranjera. Para lo cual se debe crear un grupo de WhatsApp para cada uno de los grupos de estudio o paralelo bajo parámetros y reglas establecidas por los docentes quienes se convertirán en monitores o los llamados administradores absolutos grupales para el control y asignación de actividades a desarrollarse en esta aplicación y la participación de todos los integrantes de los grupos de estudio.

Entre las actividades propuestas está el uso de canciones y frases célebres, memes, imágenes mensajes con contenido educativo y que será proporcionado por el investigador con las instrucciones y tareas claras las cuales deben estar enfocadas al aprendizaje de palabras nuevas para incrementar el vocabulario de los estudiantes de cada uno de los grupos de WhatsApp. 


\section{Metodologia.}

\section{Participantes}

Este estudio estuvo dirigido a 69 estudiantes de la Unidad Educativa "San Gerardo" de la ciudad de Riobamba.

\section{Diseño de la Investigación}

Esta investigación es cuali - cuantitativa debido a que extraen datos de la aplicación de encuestas y se comparan resultados del Pre y Post Test utilizados como instrumentos de medición. El diseño es cuasi - experimental, ya que el investigador manipula la variable dependiente (actividades colaborativas a través de WhatsApp) para medir su efecto en la variable dependiente (Incremento de vocabulario en inglés), los grupos (población) se asignaron antes del experimento y la investigación tuvo lugar en un escenario educativo donde no hay un control experimental completo. Este trabajo de investigación es transversal ya que tuvo lugar en un cierto período de tiempo del año escolar 2016-2017 utilizando una encuesta para recopilar información. Es también correlacional porque relaciona las variables involucradas en la investigación.

\section{Instrumentos y Técnicas de Recolección de datos.}

Los instrumentos aplicados en la presente investigación son encuestas y pruebas. La encuesta se aplica a toda la población y cuenta con 10 preguntas enfocadas al uso de dispositivos móviles en el aprendizaje de vocabulario.

El aprendizaje de vocabulario es crucial para dominar estructuras gramaticales. La importancia del vocabulario no se debe limitar a una lista de palabras que los estudiantes deben memorizar (Lebrón, 2009).

David Wilkins (1972) establece con mucha razón que "Aunque sin gramática poco puede ser transmitido, sin vocabulario nada puede ser transmitido". Por tanto, es necesario entender que las personas integran nuevo vocabulario a su léxico de manera gradual y que deben reconocer una palabra antes de incorporarla a su vocabulario (Wiky Estudiantes,Org., 2014).

Es así que, la presente investigación plantea la siguiente interrogante: ¿En qué medida la implementación de actividades colaborativas a través de WhatsApp incrementa el vocabulario en inglés?

\section{Plan de Análisis de datos.}

Para el análisis de datos se combinan la estadística descriptiva e inferencial. En las encuestas se utilizaron tablas en Excel y gráficos de pastel acompañados del respectivo análisis e 
interpretación de datos. En cuanto a los resultados de las pruebas se aplica el software estadístico SPSS (Statistical Package for Social Science) a través de la Prueba T.

\section{Procedimiento.}

La investigación se desarrolla en tres fases fundamentales. La primera fase responde a la planificación de actividades y elección de documentación para definir las categorías principales del marco conceptual y las dimensiones de cada una.

Dentro de las actividades de la primera fase se incluyen el trámite para solicitar la autorización de la directora de la Unidad Educativa para poder llevar a cabo la investigación, así como la firma de una carta de consentimiento de parte de los estudiantes quienes manifiestan estar dispuestos a formar parte de esta investigación, la misma que se desarrolla en las horas asignadas para la materia de inglés con la participación activa del docente encargado de la misma. En la segunda fase, se aplican las encuestas a los involucrados una vez que las preguntas y contenido de las mismas han sido validadas por dos expertos en el área al igual que las pruebas. Durante la segunda fase se ponen en práctica actividades colaborativas a través de WhatsApp, previa la ejecución de un taller sobre el uso de esta aplicación para mejorar el vocabulario en inglés. En la tercera fase se realiza el análisis e interpretación de resultados, tabulando los datos obtenidos de manera exhaustiva considerando las dimensiones e indicadores de las variables dependiente e independiente.

\section{Resultados.}

Los resultados se desarrollaron en tres fases:

\section{Primera Fase:}

La tabla 1 muestra el tamaño de la población y sus características de género Hombre / Mujer; que permitieron trabajar con todo el universo como muestra para obtener resultados más confiables.

Tabla 1. Población

\begin{tabular}{llll}
\hline Población & Mujeres & Hombres & Total \\
\hline 1ro & 15 & 10 & 25 \\
2do & 19 & 6 & 25 \\
3ro & 12 & 7 & 19 \\
& & TOTAL & $\mathbf{6 9}$ \\
\hline
\end{tabular}

Fuente: Unidad Educativa San Gerardo 
La tabla 2 presenta la encuesta con un banco de 10 preguntas que fue aplicada a los estudiantes de Bachillerato General Unificado a fin de conocer sus opiniones sobre el uso de dispositivos móviles y aplicaciones para el mejoramiento de vocabulario en el proceso de enseñanza aprendizaje del idioma inglés.

Tabla 2. Encuesta Estudiantes 1ro, 2do, y 3ro B.G.U.

\section{Encuesta}

\begin{tabular}{|c|c|c|}
\hline Pregunta & Opciones & Frec. \\
\hline \multirow{2}{*}{$\begin{array}{l}\text { Pregunta } \mathbf{N}^{\circ} \mathbf{1} \\
\text { ¿Tiene usted acceso a dispositivos móviles? }\end{array}$} & $\mathrm{Si}$ & 60 \\
\hline & No & 9 \\
\hline \multirow{2}{*}{$\begin{array}{l}\text { Pregunta } \mathbf{N}^{\circ} \mathbf{2} \\
\text { ¿Es usted propietario de un dispositivo móvil? }\end{array}$} & $\mathrm{Si}$ & 55 \\
\hline & No & 14 \\
\hline Pregunta $\mathrm{N}^{\circ} 3$ & Laptop & 10 \\
\hline \multirow{3}{*}{$\begin{array}{l}\text { ¿De los dispositivos móviles aquí nombrados cual es el } \\
\text { que más utiliza? }\end{array}$} & Tableta & 5 \\
\hline & Celular & 45 \\
\hline & Ninguna & 9 \\
\hline \multirow{4}{*}{$\begin{array}{l}\text { Pregunta } \mathbf{N}^{\circ} \mathbf{4} \\
\text { ¿Cuál es la actividad que más desarrolla con su dispositivo } \\
\text { móvil? }\end{array}$} & Llamadas & 40 \\
\hline & Estudio & 0 \\
\hline & Video & 9 \\
\hline & Redes Sociales & 20 \\
\hline \multirow{4}{*}{$\begin{array}{l}\text { Pregunta } \mathbf{N}^{\circ} \mathbf{5} \\
\text { ¿Qué tiempo promedio al día usted considera que hace uso } \\
\text { de su dispositivo móvil? }\end{array}$} & Menos 1 Hora & 9 \\
\hline & 1 a 2 Horas & 5 \\
\hline & 2 a 3 Horas & 45 \\
\hline & 3 o más Horas & 10 \\
\hline \multirow{2}{*}{$\begin{array}{l}\text { Pregunta } \mathbf{N}^{\circ} \mathbf{6} \\
\text { ¿Alguna vez ha utilizado en su dispositivo móvil aplica- } \\
\text { ciones con fines académicos? }\end{array}$} & $\mathrm{Si}$ & 10 \\
\hline & No & 59 \\
\hline \multirow{2}{*}{$\begin{array}{l}\text { Pregunta } \mathbf{N}^{\circ} 7 \\
\text { ¿Ha utilizado en su dispositivo móvil aplicaciones para es- } \\
\text { tudiar inglés? }\end{array}$} & $\mathrm{Si}$ & 2 \\
\hline & No & 6 \\
\hline \multirow{2}{*}{$\begin{array}{l}\text { Pregunta } \mathbf{N}^{\circ} \mathbf{8} \\
\text { ¿Desearía utilizar en su dispositivo móvil una aplicación } \\
\text { para mejorar su vocabulario (Léxico) en inglés? }\end{array}$} & $\mathrm{Si}$ & 66 \\
\hline & No & 3 \\
\hline \multirow{2}{*}{$\begin{array}{l}\text { Pregunta } \mathbf{N}^{\circ} \mathbf{9} \\
\text { ¿Le gustaría utilizar dispositivos móviles para el proceso } \\
\text { enseñanza-aprendizaje del inglés? }\end{array}$} & $\mathrm{Si}$ & 67 \\
\hline & No & 2 \\
\hline
\end{tabular}


Pregunta $\mathrm{N}^{\circ} 10$

$\mathrm{Si} \quad 64$

¿Está usted de acuerdo en utilizar diccionarios con aplicaciones móviles para ampliar su vocabulario y encontrar el No significado de nuevo vocabulario en inglés?

Fuente: Unidad Educativa San Gerardo

En la pregunta 1, los resultados muestran que el $87 \%$ de estudiantes tienen acceso a dispositivos móviles, mientras que en la pregunta 2 , el 79,7 \% de estudiantes es propietario de un dispositivo móvil y finalmente en la pregunta 3 el $65,2 \%$ coincide en que el dispositivo móvil más utilizado son los celulares; lo cual beneficia a que los estudiantes y docentes interactúen libremente utilizando el celular como herramienta de aprendizaje.

En la pregunta 4, el 58\% de estudiantes utiliza el celular para realizar llamadas, el $29 \%$ lo usan en redes sociales y $0 \%$ utilizan sus celulares para propósitos educativos. En la pregunta 5 , el $65,2 \%$ de estudiantes expresan que usan celulares en un promedio de 2 a 3 horas diarias.

Por otra parte, los resultados de la pregunta 6, indican que el 85,5\% de estudiantes no han utilizado aplicaciones móviles, lo cual permite apreciar que tienen muy poca experiencia en el uso de aplicaciones académicas. La pregunta 7, revela que el 97,1\% de estudiantes tiene poco conocimiento en uso de dispositivos móviles con aplicaciones para estudiar inglés; pero también se constató en la pregunta 8 , que un 95,7\% de estudiantes desearían utilizar una aplicación para mejorar vocabulario.

En la pregunta 9, los resultados demuestran que $97,1 \%$ de estudiantes utilizarían dispositivos móviles para aprender inglés y finalmente en la pregunta 10, el 92,8\% de estudiantes están de acuerdo en utilizar diccionarios con aplicaciones móviles.

Se concluye que los estudiantes están interesados en el uso de dispositivos móviles y aplicaciones para el proceso de enseñanza-aprendizaje de inglés; utilizando nuevos enfoques, metodologías y técnicas para mejorar vocabulario.

\section{Segunda Fase:}

En esta fase el Pre y Post Test propuesto permitieron establecer una relación entre las variables de la investigación siendo factores importantes a considerar en "un antes" y "un después."

La tabla 3 presenta los resultados obtenidos en las pruebas, mostrando los efectos del uso de WhatsApp en los teléfonos celulares 
Tabla 3. Tabla Comparación promedios del Pre y Post Test.

\begin{tabular}{lll}
\hline Curso & Pre Test & Post Test \\
\hline 1ro & 6,3 & 7,9 \\
2do & 5,8 & 8,1 \\
3ro & 6,4 & 8,2 \\
\hline
\end{tabular}

Fuente: Unidad Educativa San Gerardo

El 1ro B.G.U. presenta un promedio de 6, 3 en el Pre Test y un promedio de 7, 9 en el Post Test. El 2do B.G.U. muestra un promedio de 5, 8 en el Pre Test y el promedio de 8, 1 en el Post Test. Finalmente, el 3ro B.G.U. indica un promedio de 1, 8 en el Pre Test y un promedio de 2, 3 en el Post Test. Estos promedios diferenciales del Pre y Post Test muestran mejoramiento en vocabulario.

\section{Tercera Fase:}

En la tabla 4, se observa el gráfico realizado en el software SPSS y el ANOVA (Análisis de varianza) para muestras pareadas para comparar y establecer las diferencias de las variables poblacionales. La prueba ANOVA mostró como factor principal que el género de la población no afectó en los resultados obtenidos.

Tabla 4. Análisis de variancia para muestras pareadas

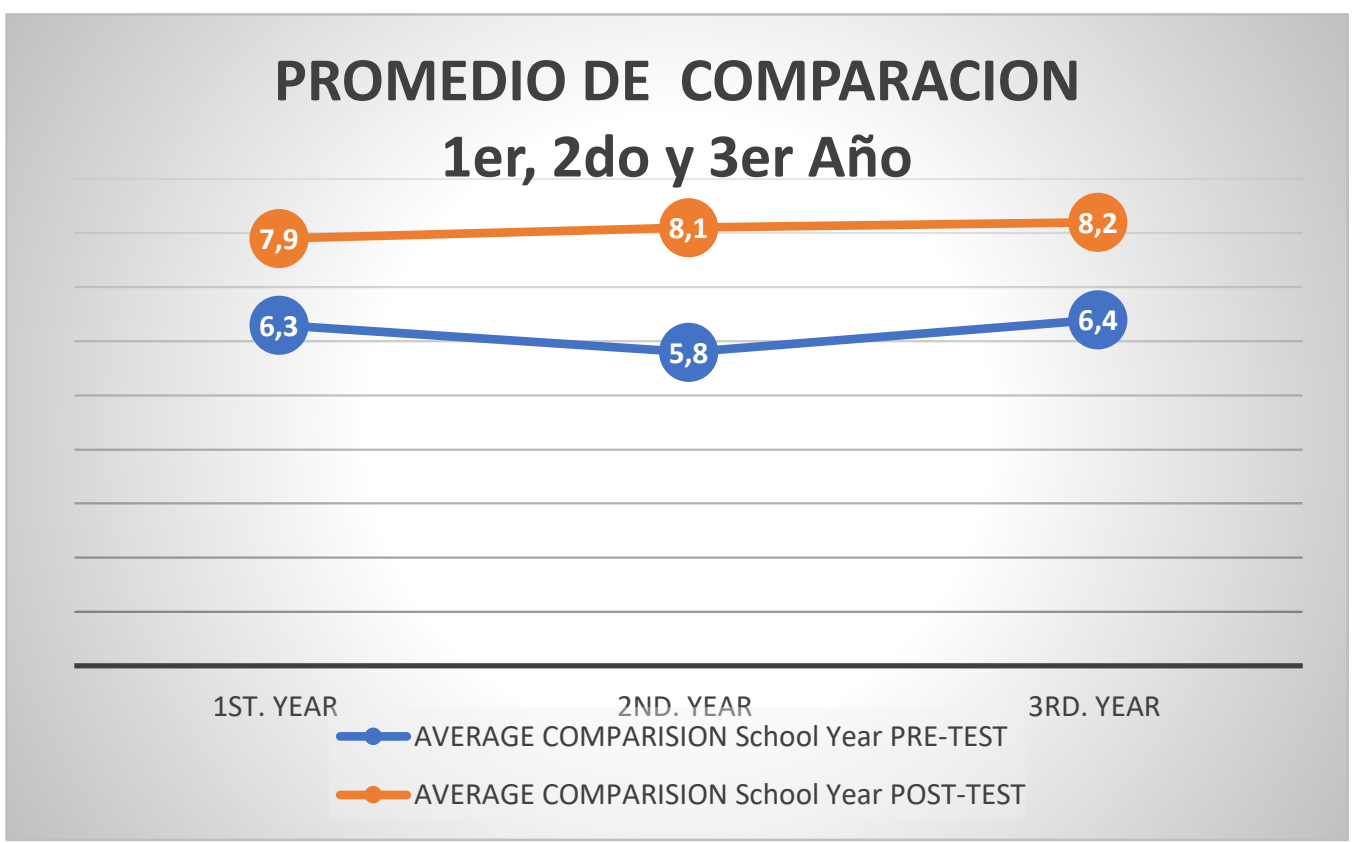

Fuente: Software SPSS 
Al mismo tiempo se aplicó la prueba T, como se divisa en la Tabla 5 para probar la hipótesis de estudio

Tabla 5. T-Test de Comparación para muestras emparejadas.

T - TEST FOR TWO PAIRED SAMPLES.

\begin{tabular}{lll}
\hline & Variable 1 & Variable 2 \\
\hline Media & 6,1304 & 8,0145 \\
Varianza & 0,103333 & 0,023333 \\
Observaciones & 3,000000 & 3,000000 \\
Coeficiente de correlación de Pearson & $-0,033942$ & \\
Diferencia hipotética de las medias & 0,000000 \\
Grados de libertad & 2,000000 \\
Estadístico t & $-9,127305$ \\
P(T<=t) una cola & 0,005896 \\
Valor crítico de t (una cola) & 2,919986 & \\
P(T<=t) dos colas & 0,011792 & \\
Valor crítico de t (dos colas) & 4,302653 & \\
\hline
\end{tabular}

Fuente: Software SPSS

La Tabla 5 muestra que el promedio en el Pre Test es de 6, 13. Sin embargo, luego de aplicar las diferentes actividades, estrategias y enfoque para promover la mejora del vocabulario obtuvieron un promedio de 8,01 . Esto es 1,9 puntos más; lo que significa que hubo un mejoramiento significativo. Se concluye que el uso adecuado de dispositivos móviles y WhatsApp si ayuda al mejoramiento de vocabulario.

\section{Discusión}

El propósito de este estudio fue determinar si la implementación de actividades colaborativas utilizando WhatsApp en dispositivos móviles incrementa la adquisición de vocabulario en inglés en los estudiantes de Bachillerato General Unificado de la Unidad Educativa "San Gerardo".

De esta manera, basado en los datos cuali - cuantitativos (Tabla 3) se determinó que los tres grupos que participaron en este estudio si tuvieron cambios considerables en tres escenarios muy puntuales: antes, durante y después del proceso de intervención.

La intervención se realizó utilizando la aplicación WhatsApp. Esta aplicación permitió crear grupos de estudio, uno por cada clase incluido el docente para el control de la participación en el lenguaje empleado y monitoreo de las actividades realizadas. Las actividades jugaron un rol muy importante en el desarrollo de nuevo vocabulario pues los estudiantes tuvieron la 
oportunidad de practicar y trabajar pues WhatsApp permite la divulgación de textos, imágenes, videos, sonido y artículos de internet enlazados (UNIVERSIA, 2016).

Antes de la intervención. Este escenario permitió obtener resultados relevantes de la encuesta aplicada y a la vez este estudio despertó el interés académico en los estudiantes.

La diferencia entre este estudio y otros estudios se centra principalmente en los resultados obtenidos en el trabajo colaborativo. Es así, que Santiago Moll (2014) declara que las actividades de aprendizaje colaborativo defienden la ayuda mutua, el apoyo entre compañeros, la heterogeneidad entre miembros de un mismo grupo y la inclusión de una Escuela de todos y para todos (Moll, 2014).

Lo que marcó la diferencia al inicio de la intervención fue que los estudiantes estaban acostumbrados a realizar actividades monótonas que se concentraban más en el uso tradicional de diccionarios físicos para el aprendizaje del nuevo vocabulario (Prado, 2015). Mientras que el uso de WhatsApp permitió una participación dinámica, profundizando la localización de palabras clave.

Durante la intervención. Se aplicó el trabajo colaborativo al formar equipos, grupos o parejas de trabajo, esto permitió compartir sentimientos, emociones y experiencias para ampliar la responsabilidad de los estudiantes. En los grupos de WhatsApp se propusieron actividades que permitieron compartir información, fotografías, vocabulario y materiales entre compañeros y profesor. Además, los estudiantes grabaron audios y videos. También, constituyó un canal de comunicación directa con el profesor para notificar trabajos, comentarios y aspectos más personales.

Pero también se presentaron ciertas limitaciones como disminuir el tiempo de otras actividades de los alumnos en el aula y la no aceptación de algunos profesores en este proceso por desconocimiento de la tecnología.

WhatsApp ofreció una experiencia real digital como herramienta para mejorar el vocabulario, el maestro se convirtió en guía y facilitador y los estudiantes fueron los protagonistas de su propio aprendizaje (Vázquez, 2016).

Después de la intervención. Se apreció que los estudiantes usan WhatsApp mucho más de lo que en realidad se imaginan, existen cientos de aplicaciones, métodos o herramientas que influyen en la adquisición de vocabulario, pero WhatsApp contribuyó a lograr el objetivo de transformar la enseñanza-aprendizaje de vocabulario en los estudiantes de 1ro, 2do, y 3ro año Bachillerato General Unificado de la Unidad Educativa "San Gerardo".

\section{Conclusiones.}


- Dentro de los resultados obtenidos y al comparar los promedios del Pre y Post Test aplicados se destacan la adquisición de nuevo vocabulario en los estudiantes de Bachillerato General Unificado de la Unidad Educativa "San Gerardo", donde se refleja en forma cuantitativa y de forma objetiva el aprendizaje de léxico en inglés como lengua extranjera.

- Categóricamente, este estudio demostró que la aplicación WhatsApp está formando parte de la vida diaria de los estudiantes y es la aplicación más utilizada en sus vidas diarias y con una guía didáctica y control adecuado se la puede utilizar con fines académicos y en este caso de estudio se utilizó para el incremento de vocabulario.

\section{Recomendaciones:}

Se debe tener en cuenta también algunos factores que pudieron afectar el resultado de la investigación, como son las limitaciones de internet y la posibilidad de que los estudiantes hicieran uso de otras aplicaciones al mismo tiempo lo cual se viene a convertir en un distractor potencial al momento de usar WhatsApp.

Sin embargo, la evidencia presentada en este estudio sobre el uso de WhatsApp para la adquisición de vocabulario en los estudiantes invita a explorar más a fondo el fenómeno del uso de los dispositivos móviles, redes sociales, aplicaciones y su posible impacto en la educación.

\section{Referencias bibliográficas.}

- Arboleda, M. (18 de Diciembre de 2017). Estadísticas de redes sociales en Ecuador 2017. Obtenido de BRANDEC: http://www.hablemosdemarcas.com/estadisticas-deredes-sociales-en-ecuador-2017/

- Basantes, A. (2017). Formación universitaria. Obtenido de Los Dispositivos Móviles en el Proceso de Aprendizaje de la Facultad de Educación Ciencia y Tecnología de la Universidad Técnica del Norte de Ecuador: https://scielo.conicyt.cl/scielo.php?script=sci_abstract\&pid=S0718$50062017000200009 \& \operatorname{lng}=\mathrm{es} \& n \mathrm{~mm}=\mathrm{iso}$

- Caldero, R. (15 de Mayo de 2014). Obtenido de El uso del teléfono móvil y la aplicación WhatsApp para el aprendizaje de verbos frasales ingleses en alumnos de nivel intermedio. España: Universidad Internacional de la Rioja.: https://reunir.unir.net/bitstream/handle/123456789/2639/caldero\%20gene.pdf?seque nce $=1 \&$ isAllowed $=\mathrm{y}$

- Calero, M. (2014). El discurso del WhatsApp. Obtenido de https://s3.amazonaws.com/academia.edu.documents/35650265/Calero._Oralia_17.p df?response-contentdisposition=inline \%3B\%20filename\%3DEl_discurso_del_WhatsApp_entre_el_Mes sen.pdf\&X-Amz-Algorithm=AWS4-HMAC-SHA256\&X-AmzCredential=AKIAIWOWYYGZ2Y53UL3A\%2F201911 
- Lebrón, A. (3 de Julio de 2009). La Enseñanza De Vocabulario En El Aula DE. Obtenido de Temas para la Educación: https://www.feandalucia.ccoo.es/andalucia/docu/p5sd5037.pdf

- Lenin Lara, M. A. (2017). Uso didáctico de WhatsApp para desarrollar la participación de los estudiantes en el. Revista Dilemas Contemporáneos: Educación, Política y $\quad$ Valores., $19 . \quad$ Obtenido de http://files.dilemascontemporaneoseducacionpoliticayvalores.com/200003715c4c06c5bf2/18.1.17\%20Uso\%20did\%C3\%A1ctico\%20de\%20WhatsApp\%20para\% 20desarrollar\%20la.....pdf

- Moll, S. (19 de Enero de 2014). ¿En qué consiste la actividad de aprendizaje cooperativo Emojiónate? Obtenido de https://justificaturespuesta.com/actividad-deaprendizaje-cooperativo-emojionate/

- Prado, J. (2015). Estrategias Y Actividades Para El Uso Del Diccionario En El Aula. Obtenido de file:///C:/Users/NEGRO/Downloads/4655Texto\%20del\%20art\%C3\%ADculo-6955-1-10-20121207.pdf

- RAMÍREZ, M. A. (2017). Aprendizaje Colaborativo A Través De Whatsapp Y Facebook. Congreso Nacional de Investigacón educativa, 9.

- Recursos Educativos. (2016). ¿El Móvil En El Aula? Ideas, Ventajas, Retos Y Posibilidades. Obtenido de Inspira TICs: https://www.inspiratics.org/es/recursoseducativos/el-movil-en-el-aula-ideas-ventajas-retos-y-posibilidades

- Rubio, J. (7 de Junio de 2015). El fenómeno WhatsApp en el contexto de la comunicación personal: una aproximación a través de los jóvenes universitarios. $\begin{array}{llll}\text { Obtenido de } & \text { ICONO }\end{array}$ https://www.icono14.net/ojs/index.php/icono14/article/view/818

- Telégrafo. (29 de Julio de 2017). Whats App alcanza los 1.000 millones de usuarios a diario. pág. $1 . \quad$ Obtenido de https://www.eltelegrafo.com.ec/noticias/tecnologia/1/whatsapp-alcanza-los-1-000millones-de-usuarios-a-diario

- UNIVERSIA. (26 de Jukio de 2016). WhatsApp para fines educativos: recomendaciones para docentes y estudiantes. Obtenido de https://noticias.universia.pr/educacion/noticia/2016/07/26/1142129/whatsapp-fineseducativos-recomendaciones-docentes-estudiantes.html

- Vázquez, F. (5 de Octubre de 2016). Whatsapp como herramienta de Marketing Digital. Obtenido de https://br.escueladenegociosydireccion.com/business/whatsappy-marketing-digital/

- Wiky Estudiantes. Org. (Enero de 21 de 2014). Por Qué Es Importante El Vocabulario En Una Lengua Extranjera? Obtenido de https://www.wikiestudiantes.org/por-quees-importante-el-vocabulario-en-una-lengua-extranjera/

\section{U Ciencia}


PARA CITAR EL ARTÍCULO INDEXADO.

Rojas Yumisaca, W., Chamorro Ortega, C., \& Bejarano Criollo, S. (2019). Implementación de actividades colaborativas a través de WhatsApp para incrementar vocabulario en inglés. Ciencia Digital, 3(4.1), 84-97. https://doi.org/10.33262/cienciadigital.v3i4.1.983

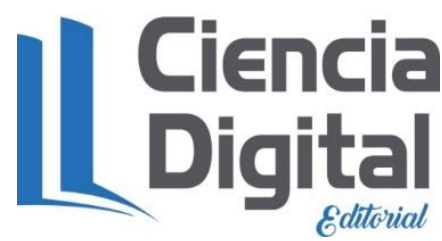

El artículo que se publica es de exclusiva responsabilidad de los autores y no necesariamente reflejan el pensamiento de la Revista Ciencia Digital.

El artículo queda en propiedad de la revista y, por tanto, su publicación parcial y/o total en otro medio tiene que ser autorizado por el director de la Revista Ciencia Digital.
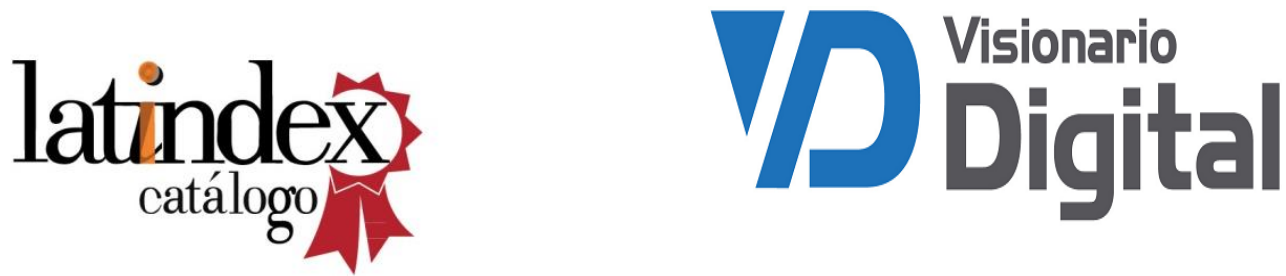